\title{
MODELING RESERVOIR WATER RELEASE DECISION USING ADAPTIVE NEURO FUZZY INFERENCE SYSTEM
}

\author{
${ }^{1}$ Suriyati Abdul Mokhtar, ${ }^{2}$ Wan Hussain Wan Ishak \& \\ ${ }^{3}$ Norita Md Norwawi \\ ${ }^{1 \& 2}$ Universiti Utara Malaysia, Malaysia \\ Universiti Sains Islam Malaysia, Malaysia
}

suriyati87@gmail.com; hussain@uum.edu.my; norita@usim.edu.my

\begin{abstract}
Reservoir water release decision is one of the critical actions in determining the quantity of water to be retained or released from the reservoir. Typically, the decision is influenced by the reservoir inflow that can be estimated based on the rainfall recorded at the reservoir's upstream areas. Since the rainfall is recorded at several different locations, the use of temporal pattern alone may not be appropriate. Hence, in this study a spatial temporal pattern was used to retain the spatial information of the rainfall's location. In addition, rainfall recorded at different locations may cause fuzziness in the data representation. Therefore, a hybrid computational intelligence approach, namely the Adaptive Neuro Fuzzy Inference System (ANFIS), was used to develop a reservoir water release decision model. ANFIS integrates both the neural network and fuzzy logic principles in order to deal with the fuzziness and complexity of the spatial temporal pattern of rainfall. In this study, the Timah Tasoh reservoir and rainfall from five upstream gauging stations were used as a case study. Two ANFIS models were developed and their performances were compared based on the lowest square error achieved from the simulation conducted. Both models utilized the spatial temporal pattern of the rainfall as input. The first model considered the current reservoir water level as an additional input, while the second model retained the existing input. The result indicated that the application of ANFIS could be used successfully for modeling reservoir water release decision. The first model with the additional input showed better performance with the lowest square error compared to the second model.
\end{abstract}


Keywords: ANFIS, decision modeling, fuzzy logic, hybrid computational intelligence, neural network, reservoir operation.

\section{INTRODUCTION}

A reservoir is one of the most important elements in the development of human civilization in providing a multi-purpose operation to fulfill human needs. A reservoir can be classified as a structural flood mitigation approach that aims to store water and maintain safe discharge at downstream areas (Smith \& Ward, 1998). As stated by Wan Ishak et al. (2012), a reservoir can be defined as a natural or artificial lake, or pond, where water is collected and stored for multipurpose operations such as water supply, flood control, hydropower generation, and used for agricultural and recreation a purposes.

Reservoir water release decision is one of the challenging tasks for reservoir operators in order to determine the amount of water to be stored and to be released from a reservoir (Norwawi et al., 2005; Wurbs, 1993). The reservoir capacity needs to be maintained in order to prevent downstream floods and to reduce water shortage problems in the future. In both flood and drought situations, the decisions regarding water release are made in accordance with the available water, inflows, demands, time, previous releases, etc. (Jain \& Singh, 2003). However, different reservoirs have different objectives and purposes, thus different operation rules are needed (Wan Ishak et al., 2012).

Typically, reservoir water release decision is based on upstream inflow that is observed through the magnitude of the upstream rainfall and the river water level. The total volume of rainfall may come from several gauging stations and their distances to the reservoir are varied (Mokhtar et al., 2016). Thus, rainfall observed at those gauging stations may take different time to reach the reservoir. This situation shows that there is a spatial temporal relationship hidden between each gauging station and the reservoir. Currently, there are limited studies that focus on this situation, as most of the previous studies focused on temporal relationship and the total rainfall volume (Mohan \& Revesz, 2012; Wan Ishak et al., 2012; Mokhtar et al., 2014). Thus, the spatial temporal relationship needs to be considered for modelling reservoir water release decision.

One of the well-known computational intelligence techniques used for modelling reservoir water release decision and forecasting is the Artificial Neural Network (ANN) (Nazri et al., 2013; Mokhtar et al., 2014; Wan Ishak 
et al., 2015). However, this technique suffers from poor interpretability, since it is difficult for humans to explain the practicality and logical meaning behind the learned weights of the model (Jothiprakash \& Kote, 2011; Kajornrit et al., 2013). This problem can be solved by the Adaptive Neuro Fuzzy Inference System (ANFIS). ANFIS is a hybrid intelligent system which combines the fuzzy processing of Fuzzy Logic (FL) and the learning capability of ANN.

The next section presents several related studies on ANFIS and its application on modeling reservoir operations, followed by the methodology, results and discussion. The last section presents the paper's conclusions.

\section{RELATED WORKS}

The application of ANFIS has been widely used in modeling complex reservoir operations and predictions. Chang and Chang (2006) presented a neuro fuzzy hybrid approach to develop a system for water level prediction during floods. Prediction models for reservoir management have been developed using ANFIS. In their study, two ANFIS models were developed: Model 1 with human decision as input variables; Model 2 without human decision as input variables. The result showed that the application of ANFIS could be used successfully to forecast reservoir water level for the next three hours.

The Neuro-fuzzy technique was proposed by Mehta and Jain (2009) to develop an operation policy which was to be used for multi-purpose reservoirs that were implemented easily by the operators. Three models of Fuzzy Rule Based (FRB) were developed and tested. ANFIS and Fuzzy Mamdani (FM) were used to compute the release from all the developed models. The result showed that the ANFIS-cluster provided the best performance compared to FM, but FM was more user-friendly.

Recently, Valizadeh and El-Shafie (2013) proposed multiple input fuzzifications in ANFIS to predict the dam level using different types of membership functions. The current model was compared with the traditional ANFIS by fuzzifying each type of input in the modeling system to demonstrate the behaviour of the different membership functions for each input. Three different time lags were observed with two different membership functions (MF) that were the generalized bell-shaped MF and the Gaussian MF for the two inputs, rainfall and dam level from previous days. Based on three statistical evaluations, the model that employed different types of MFs performed better than the other models, especially for time lag. 
Valizadeh et al. (2014) propounded a different ANFIS model in order to apply different types of MFs for each type of input for water level forecasting. This study was applied for two case studies: the Klang Gates Dam and the Rantau Panjang Station. Two different ANFIS models were proposed to observe the performance of the new fuzzifying pattern in the ANFIS model with different types of MFs. Based on the results, the model which applied the generalized bell-shaped MF and the Gaussian MF provided better results in the two case studies compared to the model that used the generalized bell-shaped MF for the two types of inputs. Overall, this study proved the ability of the model to apply different types of membership function in the ANFIS model.

\section{METHODOLOGY}

In this study, the Timah Tasoh reservoir was used as a case study. It is located in the state of Perlis, Malaysia. The Timah Tasoh reservoir is one of the largest multi-purpose reservoirs in Northern Peninsular Malaysia. The reservoir serves as flood mitigation and is the only reservoir which has a gate structure that involves decisions. In this study, the daily reservoir water level (WL) and rainfall (RF) from 1999-2012 were obtained from the Department of Irrigation Drainage Perlis, Malaysia. The Timah Tasoh reservoir is influenced by upstream rainfall, which is automatically recorded through five upstream gauging stations. An example of the data is shown in Table 1.

Table 1

Example of Reservoir Water Level and Rainfall Data

\begin{tabular}{|c|c|c|c|c|c|c|}
\hline \multirow[b]{2}{*}{$\begin{array}{c}\text { Reservoir } \\
\text { water level (m) }\end{array}$} & \multicolumn{5}{|c|}{ Rainfall (mm) } & \multirow[b]{2}{*}{ Gate } \\
\hline & $\begin{array}{c}\text { Padang } \\
\text { Besar }(p b)\end{array}$ & $\begin{array}{c}\text { Tasoh } \\
(t s)\end{array}$ & $\begin{array}{c}\text { Lubuk } \\
\text { Sireh }(l s)\end{array}$ & $\begin{array}{c}\text { Kaki } \\
\text { Bukit } \\
(k b) \\
\end{array}$ & $\begin{array}{c}\text { Wang } \\
\text { Kelian } \\
(w k)\end{array}$ & \\
\hline 29.250 & 10.50 & 0.00 & 22.00 & 40.50 & 9.00 & Close \\
\hline 29.275 & 0.00 & 0.00 & 42.00 & 30.00 & 34.00 & Open \\
\hline 29.365 & 0.00 & 0.00 & 0.00 & 10.00 & 1.50 & Open \\
\hline 29.360 & 0.00 & 30.00 & 0.00 & 0.00 & 21.50 & Open \\
\hline 29.265 & 0.00 & 63.00 & 33.00 & 50.00 & 0.00 & Open \\
\hline 29.215 & 0.00 & 0.00 & 2.00 & 0.00 & 0.00 & Open \\
\hline 29.145 & 0.00 & 0.00 & 0.00 & 0.00 & 0.00 & Open \\
\hline
\end{tabular}




\begin{tabular}{ccccccc}
\hline & \multicolumn{5}{c}{ Rainfall $(\mathbf{m m})$} & \\
\cline { 2 - 5 } $\begin{array}{c}\text { Reservoir } \\
\text { water level }(\mathbf{m})\end{array}$ & $\begin{array}{c}\text { Padang } \\
\text { Besar }(\boldsymbol{p b})\end{array}$ & $\begin{array}{c}\text { Tasoh } \\
(\boldsymbol{t} \boldsymbol{t})\end{array}$ & $\begin{array}{c}\text { Lubuk } \\
\text { Sireh }(\boldsymbol{l s})\end{array}$ & $\begin{array}{c}\text { Kaki } \\
\text { Bukit } \\
(\boldsymbol{k b})\end{array}$ & $\begin{array}{c}\text { Wang } \\
\text { Kelian } \\
(\boldsymbol{w} \boldsymbol{k})\end{array}$ & Gate \\
\hline 29.035 & 0.00 & 0.00 & 0.00 & 10.00 & 0.00 & Open \\
29.005 & 0.00 & 0.00 & 0.00 & 20.00 & 0.00 & Close \\
29.000 & 0.00 & 0.00 & 0.00 & 0.00 & 6.50 & Close \\
29.000 & 18.50 & 0.00 & 0.00 & 10.00 & 1.50 & Close \\
28.995 & 0.00 & 0.00 & 0.00 & 6.00 & 0.00 & Close \\
28.995 & 30.00 & 0.00 & 0.00 & 0.00 & 0.00 & Close \\
28.990 & 0.00 & 0.00 & 3.00 & 8.00 & 0.00 & Close \\
28.995 & 0.00 & 16.00 & 21.00 & 0.00 & 0.00 & Close \\
\hline
\end{tabular}

Figure 1 shows the location of the Timah Tasoh reservoir and the five gauging stations: Padang Besar ( $p b)$, Tasoh ( $t s)$, Lubuk Sireh $(l s)$, Kaki Bukit $(k b)$, and Wang Kelian $(w k)$. The gauging stations are located at different locations and the distance between the gauging stations and the reservoir are varied (Mokhtar et al., 2016). Mokhtar et al. identified the spatial temporal relationship between the Timah Tasoh reservoir and the upstream gauging stations. Based on their study, the rainfall recorded at $p b$ and $w k$ took 6 days to affect the Timah Tasoh reservoir water level. The rainfall recorded at $t s$ and $k b$ took 3 days while the rainfall recorded at $l s$ took 4 days to affect the Timah Tasoh reservoir water level. These values were adopted in this study.

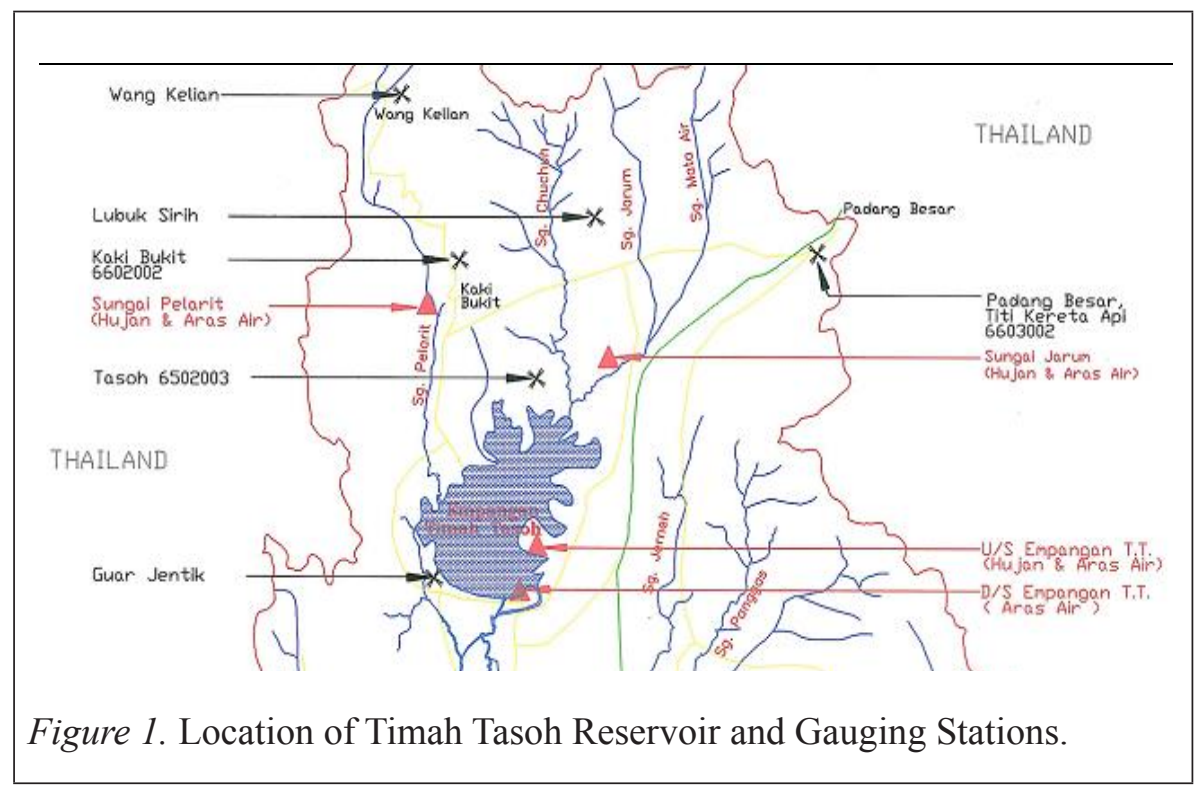


In this study, the temporal patterns of rainfalls from each gauging station were mapped with the reservoir gate opening events: open or close gates. After the selection, only 249 data were used and the data were divided into three datasets: training (70\%), validation (15\%), and testing sets (15\%). The training set comprised of 173 datasets; the validation set had 38 datasets; while the remaining were allocated for the testing set (38 datasets). Table 2 shows the selection of the data from the actual datasets based on gate opening events, either open or close.

Table 2

Selection of Datasets

\begin{tabular}{cccc}
\hline Datasets & Training & Validation & Testing \\
\hline Actual data & 3536 & 758 & 758 \\
Open or close gate & 173 & 38 & 38 \\
\hline
\end{tabular}

In this study, ANFIS was used for the modeling reservoir water release decision. Fundamentally, ANFIS can be defined as a network model representation of the Sugeno-type fuzzy system, whose MF parameters are adjusted using either a back propagation algorithm alone, or in combination with a least square type of method (Jang, 1993). A first order Sugeno-type fuzzy model has the following rules (Cruz \& Mestrado, 2009):

$$
\begin{aligned}
& \text { Rule 1: If } x \text { is } A_{1} \text { and } \mathrm{y} \text { is } B_{1} \text {, then } f_{1}=p_{1} x+q_{1} y+r 1 \\
& \text { Rule 2: If } x \text { is } A_{2} \text { and } \mathrm{y} \text { is } B_{2} \text {, then } f_{2}=p_{2} x+q_{2} y+r 2
\end{aligned}
$$

Figure 2 shows the ANFIS architecture for two inputs of the Sugeno fuzzy model with four rules. This architecture consists of five layers: Layer 1 - input nodes; Layer 2 - rules nodes; Layer 3 - average nodes; Layer 4 - consequent nodes; and Layer 5 - output nodes.

In this study, two ANFIS models were developed for gate opening decisions: Model 1 and Model 2. The input and output of the models are represented as follows:

Model 1 consisted of 23 input variables (temporal rainfall patterns from five upstream gauging stations and the current reservoir water level):

$G o(t+i)=R F(p b(t-5), p b(t-4), p b(t-3), p b(t-2), p b(t-1), p b$ $(t), t s(t-2)$, 
ts (t-1), ts (t), ls (t-3), ls (t-2), ls (t-1), ls (t), $k b(t-2), k b(t-1), k b$

$(t), w k(t-5), w k(t-4), w k(t-3), w k(t-2), w k(t-1), w k(t)), W L(t)$
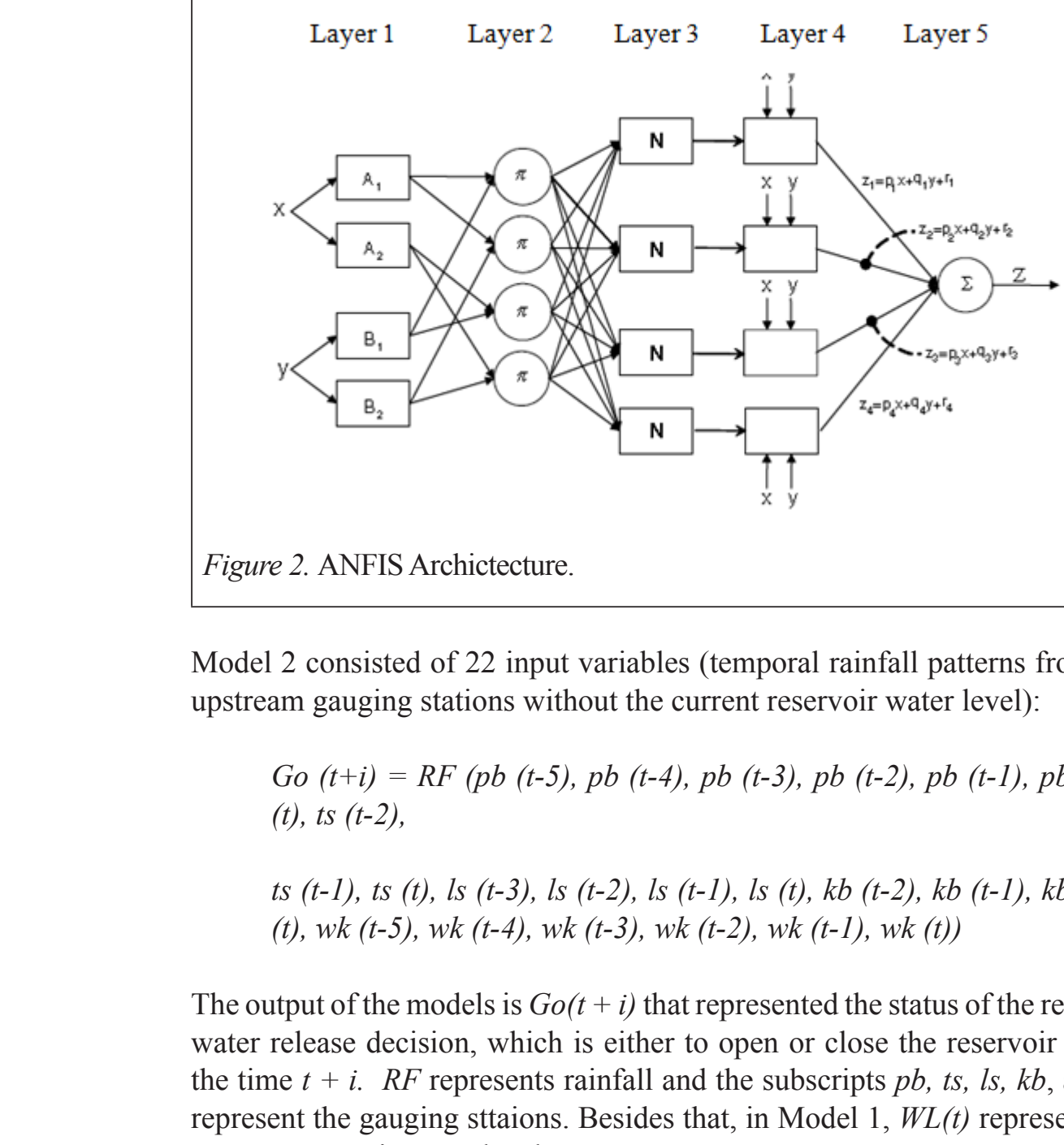

Figure 2. ANFIS Archictecture.

Model 2 consisted of 22 input variables (temporal rainfall patterns from five upstream gauging stations without the current reservoir water level):

$G o(t+i)=R F(p b(t-5), p b(t-4), p b(t-3), p b(t-2), p b(t-1), p b$

$(t), t s(t-2)$,

ts (t-1), ts (t), ls (t-3), ls (t-2), ls (t-1), ls (t), $k b(t-2), k b(t-1), k b$

$(t), w k(t-5), w k(t-4), w k(t-3), w k(t-2), w k(t-1), w k(t))$

The output of the models is $G o(t+i)$ that represented the status of the reservoir water release decision, which is either to open or close the reservoir gate at the time $t+i . R F$ represents rainfall and the subscripts $p b, t s, l s, k b$, and $w k$ represent the gauging sttaions. Besides that, in Model 1, $W L(t)$ represents the current reservoir water level.

ANFIS was implemented in MATLAB. Table 3 shows the parameters used in the ANFIS model. The number of epochs refers to the frequency to train the model. The method of optimization refers to the optimization methods available for the fuzzy inference system training, namely hybrid (the default, mixed least squares) and back propagation. Meanwhile, error tolerance refers to the value of errors that can be tolerated by the model (default $=0$ ). 

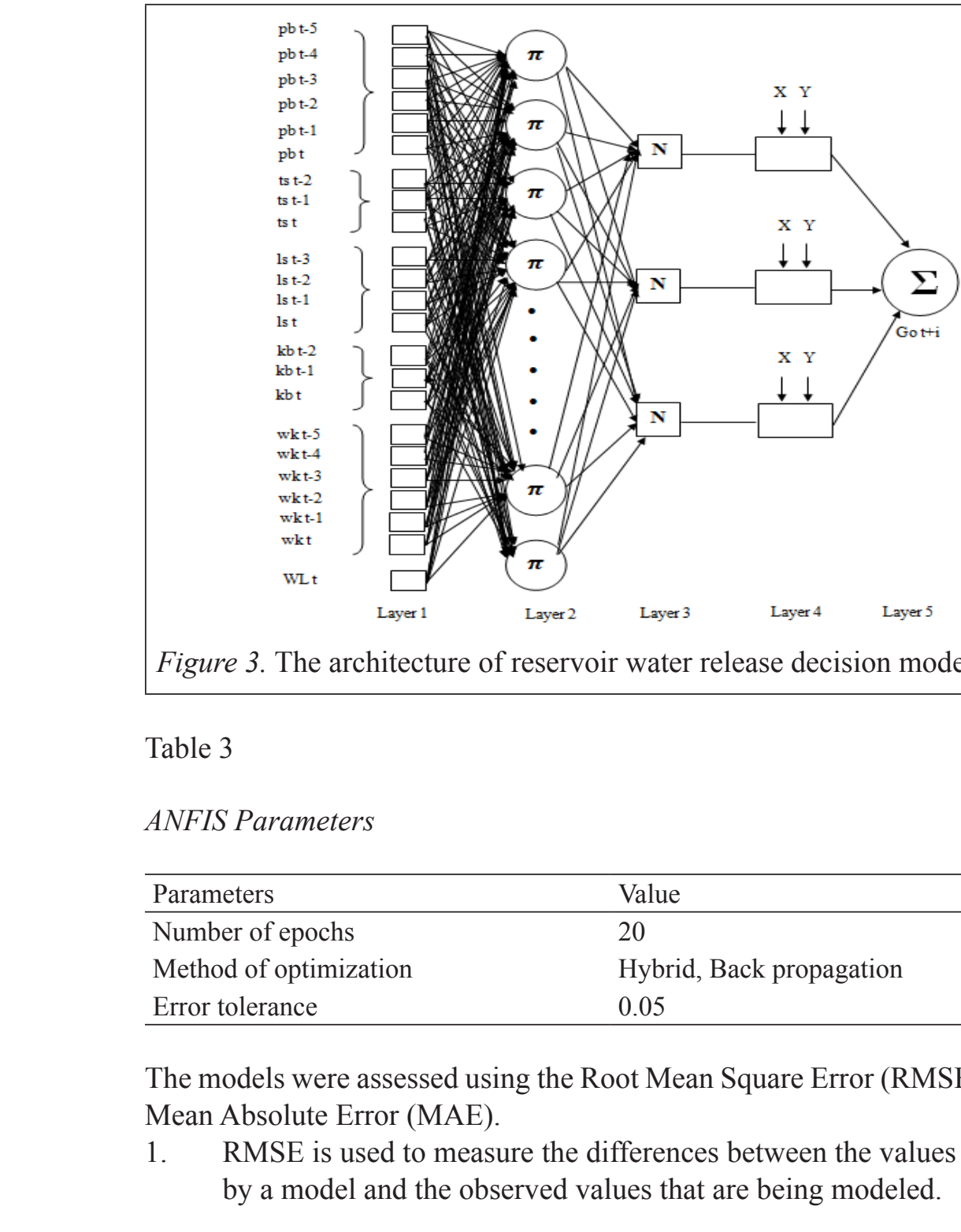

Figure 3. The architecture of reservoir water release decision model.

Table 3

\section{ANFIS Parameters}

\begin{tabular}{ll}
\hline Parameters & Value \\
\hline Number of epochs & 20 \\
Method of optimization & Hybrid, Back propagation \\
Error tolerance & 0.05 \\
\hline
\end{tabular}

The models were assessed using the Root Mean Square Error (RMSE) and the Mean Absolute Error (MAE).

1. RMSE is used to measure the differences between the values predicted by a model and the observed values that are being modeled.

$$
\text { RMSE }=\sqrt{\frac{\sum_{\mathrm{i}=1}^{\mathrm{n}}\left(\mathrm{X}_{\mathrm{obs,i}}-\mathrm{X}_{\text {model } \mathrm{j}}\right)^{2}}{\mathrm{n}}}
$$

2. MAE measures the average of the absolute error and if the lower value is better.

$$
\text { MAE }=\frac{1}{n} \sum_{i=1}^{n}\left|f_{i}-y_{i}\right|=\frac{1}{n} \sum_{i=1}^{n}\left|e_{i}\right|
$$




\section{RESULTS AND DISCUSSION}

Table 4 shows the results for both Model 1 and Model 2 for training, checking and testing sets. The performances of the models are measured using two performance criteria; RMSE and MAE. RMSE and MAE have been calculated using Equations (1) and (2).

Table 4

Result for ANFIS Models

\begin{tabular}{llllllllll}
\hline \multirow{2}{*}{$\begin{array}{c}\text { ANFIS } \\
\text { model }\end{array}$} & Method & Input & \multicolumn{3}{c}{ RMSE } & \multicolumn{3}{c}{ MAE } \\
\cline { 4 - 9 } & & & \multicolumn{2}{c}{ Training } & Validation & Testing & Training Validation & Testing \\
\hline \multirow{2}{*}{ Model 1 } & Hybrid & 23 & 0.37408 & 0.62738 & 0.37408 & 0.38246 & 0.66845 & 0.38246 \\
& Back propa & 23 & 0.83175 & 0.65645 & 0.45022 & 0.43847 & 0.71756 & 0.72843 \\
& & & & & & & & \\
\multirow{2}{*}{ Model 2 } & Hybrid & 22 & 0.41391 & 0.66190 & 0.41391 & 0.41486 & 0.69457 & 0.41486 \\
& Back propa & 22 & 0.62303 & 0.71281 & 0.59164 & 0.45392 & 0.58141 & 0.50492 \\
\hline
\end{tabular}

As mentioned earlier, two ANFIS models were developed with different numbers of input variables, which were 23 for Model 1, and 22 for Model 2. The performances of the ANFIS models were compared using three different datasets: training set, validation set, and testing set. Both Model 1 and Model 2 have been trained with different methods of optimization: hybrid and back propagation.

Based on the results in Table 4, the hybrid method performed well compared to the back propagation method. Both Model 1 and Model 2 have the lowest error rate using the hybrid method. The lowest errors achieved for training, validation and testing sets are $(0.374083,0.62738,0.37408)$ and $(0.38246$, $0.66845,0.38246$ ) for Model 1 with 23 inputs. The development of Model 1 which included the reservoir water level as an input variable had a better performance compared to Model 2. This shows that reservoir water level is one of the important factors in the reservoir water release decision. This is in line with Chang and Chang (2006).

On the whole, based on the results, it shows that the two proposed ANFIS models perform well for modeling reservoir water release decisions. The results illustrate that the application of ANFIS can be used to develop the decision models which might provide accurate water release prediction. 


\section{CONCLUSION}

In this study, a hybrid computational intelligence approach, ANFIS, was proposed for modeling reservoir water release decision. ANFIS is a powerful modeling tool because it combines the learning ability of ANN and FL. The result of this study shows that the ANFIS model which applied the hybrid method performs well compared to the back propagation method. Model 1 has a better performance compared to Model 2 with different numbers of input variables. Overall, the performance of ANFIS indicates that it can be applied successfully for modeling reservoir water release decision.

In addition, the results proved that the reservoir water level can be one of the variables that give significant effects on the water release decision. In practice, reservoir water release decision is crucial, since the decision deals with many complicated variables and multi-purpose objectives. Reservoir water release decision is one of the emergency situations that requires a fast and accurate decision. Thus, modeling reservoir water release decision is vital in order to save human lives or prevent downstream flood risks.

\section{ACKNOWLEDGMENTS}

The authors wishes to thank the Ministry of Higher Education of Malaysia for funding this study under the Long Term Research Grant Scheme (LRGS/ b-u/2012/ UUM/Teknologi Komunikasi dan Informasi) and the Department of Irrigation and Drainage Malaysia for supplying hydrology and reservoir operational data.

\section{REFERENCES}

Chang, F. J., \& Chang, Y. T. (2006). Adaptive neuro-fuzzy inference system for prediction of water level in reservoir. Advances in Water Resources, 29(1), 1-10.

Cruz,A., \& Mestrado, N. C. E. (2009). ANFIS: Adaptive neuro-fuzzy inference systems. IM, UFRJ, Mestrado NCE.

Jain, S. K., \& Singh, V. P. (2003). Chapter 11: Reservoir operation. Water Resources Systems Planning \& Management, 51, 615-679.

Jang, J. S. (1993). ANFIS: Adaptive-network-based fuzzy inference system. Systems, Man and Cybernetics, IEEE Transactions on, 23(3), 665-685. 
Jothiprakash, V., \& Kote, A. S. (2011). Improving the performance of datadriven techniques through data pre-processing for modelling daily reservoir inflow. Hydrological Sciences Journal, 56(1), 168-186.

Kajornrit, J., Wong, K. W., \& Fung, C. C. (2013). A modular technique for monthly rainfall time series prediction. In 2013 IEEE Symposium on Computational Intelligence in Dynamic and Uncertain Environments (CIDUE) (pp. 76-83).

Liu, X., Guo, S., Liu, P., Chen, L., \& Li, X. (2011). Deriving optimal refill rules for multi-purpose reservoir operation. Water Resources Management, 25(2), 431-448. doi:10.1007/s11269-010-9707-8

Mehta, R., \& Jain, S. K. (2009).Optimal operation of a multi-purpose reservoir using neuro-fuzzy technique. Water Resources Management, 23(3), 509-529. doi:10.1007/s11269-008-9286-0.

Mohan, A., \& Revesz, P. (2012).Temporal data mining of uncertain water reservoir data. Proceedings of the Third ACM SIGSPATIAL International Workshop on Querying and Mining Uncertain Spatio-Temporal Data (pp. 10-17). New York, NY, USA: ACM.

Mokhtar, S.A., Wan Ishak, W.H., \& Norwawi, N.M. (2014). Modelling of reservoir water release decision using neural network and temporal pattern of reservoir water level. Proceedings of the Fifth International Conference on Intelligent Systems, Modelling and Simulation, pp. 127130 .

Mokhtar, S.A., Wan Ishak, W.H., \& Norwawi, N.M. (2016). Investigating the spatial relationship between the upstream gauging stations and the reservoir. Journal of Advanced Management Science, 4(6), 503-506.

Nazri, N., Suliman, A., Othman, M. \& Ku-Mahmud, K. R., (2013). Artificial neural network and support vector machine in flood forecasting: A review. Proceedings of the 4th International Conference on Computing and Informatics, pp 327-333.

Norwawi, N.M., Ku-Mahamud, K..R. \& Deris, S. (2005). Recognition decision-making model using temporal data mining technique. Journal of Information and Communication Technology, 4, 37-56. 
Smith, K., \& Ward, R. (1998). Floods: Physical processes and human impacts (p. 394). Chichester: Wiley.

Valizadeh, N., \& El-Shafie, A. (2013). Forecasting the level of reservoirs using multiple input fuzzification in ANFIS. Water Resources Management, 27(9), 3319-3331. doi:10.1007/s11269-013-0349-5.

Valizadeh, N., El-Shafie, A., Mirzaei, M., Galavi, H., Mukhlisin, M., \& Jaafar, O. (2014). Accuracy enhancement for forecasting water levels of reservoirs and river streams using a multiple-input-pattern fuzzification approach. The Scientific World Journal, 2014.

Wan Ishak, W. H., Ku Mahamud, K. R., \& Md Norwawi, N. (2012). Modelling reservoir water release decision using temporal data mining and neural network. International Journal of Emerging Technology and Advanced Engineering, ISSN 2250-2459 (Online), 2(8), 422-428.

Wan Ishak, W.H., Ku-Mahamud, K.R. \& Norwawi, N.M. (2011). Mining temporal reservoir data using sliding window technique. CiiT International Journal of Data Mining Knowledge Engineering, 3(8),473-478.

Wan Ishak, W.H., Ku-Mahamud, K.R., \& Norwawi, N.M. (2015). Modelling of human expert decision making in reservoir operation. Journal Teknologi, 77(22), 1-5.

Wurbs, R.A. (1993). Reservoir-system simulation and optimization models. Journal of Water Resources Planning And Management, 119(4), 455472. 